\title{
QA/QC as a Pressing Need for Microarray Analysis: Meeting Report from CAMDA'02
}

\author{
Kim Johnson and Simon Lin \\ Duke Bioinformatics Shared Resource, Duke University Medical Center, Durham, NC, USA
}

BioTechniques 34:S62-S63 (March 2003)

\section{INTRODUCTION}

The Critical Assessment of Microarray Data Analysis (CAMDA) was held November 14-15 in Durham, NC. Sponsored by the Duke Bioinformatics Shared Resource, the conference brought together 174 participants from 11 countries to discuss microarray data analysis. As always, the conference organizers provided two published data sets (www.camda.duke.edu/camda02/contest.asp) and asked participants to analyze one of the two in a contest format to introduce new approaches to microarray data analysis. However, this year's contest ultimately became a discussion of the real world data quality problems inherent in microarrays.

The recent efforts to develop centralized expression databases (1) to catalog microarray results could potentially transform research (2). Such databases would allow us to test and generate new hypotheses in the same way that GenBank ${ }^{\circledR}$ was used for sequence analysis. However, new techniques bring up old problems. Without a rigorous quality assurance and quality control (QA/QC) procedure for microarray data, it may be premature to integrate data generated by different laboratories with different platforms. At this point, even the quality and comparability of data generated by the same laboratory over a period of time may be questionable. The lessons learned at the third CAMDA support this assessment. While many of the presentations discussed new analytical methods, the Best Presentation award went to Kevin Coombes (M.D. Anderson) for his contributions in detecting abnormalities in the CAMDA contest data set using rigorous QA algorithms before the data were analyzed for differential gene expression.

\section{A DISCUSSION OF DATA ABNORMALITIES}

At the CAMDA'02 conference, the Project Normal data set (3) served as a real world test. Many groups noticed abnormalities at different levels in the data set using either analysis of variance (ANOVA) modeling (Russ Wolfinger, SAS, and Xiangqin Cui, Jackson Lab), Bayesian analysis (M. Bhattacharjee, University of Helsinki, and M. Ochs, Fox Chase Cancer Center), or image characterization approaches (David Lalush, NC State University). Liling Warren (NC State University) described abnormalities in the testis tissue measurements. Wolfinger illustrated the abnormalities in the data set by running a simple scatter plot in SAS-JMP. Within the kidney data set, many observed a distinctly different pattern in mice five and six. Lalush pointed out that the image quality of these mice was degraded and may have caused such a discrepancy. Colin Prichard, author of the original data set, counter-argued that RT-PCR of several genes did indeed confirm that mice five and six have truly different expression patterns. Thus, the cause of the pattern deviations remains unclear.

A unique and distinctive paper was presented by the Coombes group, whose approach was to do a rigorous QA step before data analysis began. This QA step identified an annotation problem within the Project Normal data set. Coombes further supported their claims by modeling the data with independent expression evidence from the UniGene (4) database.

Coombes said that similar data issues crop up regularly and thus the QA step has been a routine procedure at M.D. Anderson. The use of clustering and principal component analysis (PCA) visualization provide a simple and effective initial data quality check. Thus, the Coombes group was able to reveal that the original CAMDA data set had errors in the annotation of both the testis and liver data. At the end of the conference, Colin Pritchard explained that the problems in the data set were a result of incorrectly merging the data with the annotations, resulting in mismatched row-column combinations. In effect, the measurements were correct, but simply mislabeled. This problem affected about one-third of the genes (though different sets) in the testis and liver data. Pritchard presented a reanalysis at the meeting using corrected data sets. According to his analysis, the results were not notably different from the original one, both in terms of quantity of genes that varied and which genes had variations. The corrected data set has now been released on the CAMDA Web site, and presenters will have the opportunity to reanalyze the data before completing their final paper for the proceedings.

Although the quality issue is more apparent in homemade cDNA arrays, measurements from Affymetrix arrays (Affymetrix, Santa Clara, CA, USA) are not immune to data quality issues. Last year, $\mathrm{Li}$ and Wong published a helpful model (5) in detecting Affymetrix image contamination. At CAMDA, Andrey Ptitsyn (Louisiana State University) showed a topological effect of the hybridization on the Affymetrix chips. He argued that this effect consistently exists on all Affymetrix chips, including human and Escherichia coli in the Affymetrix Latinsquare data set (6). Sema Kachalo (University of Illinois) and Li Zhang (M.D. Anderson) discussed cross-hybridizations on the Affymetrix chips. Russ Wolfinger showed how mixed-model ANOVA is useful for QA/QC and analysis of Affymetrix data, using perfect-match only data extracted from .CEL files. Together with the presentation by Rafael Irizarry (Johns Hopkins) on probe-level Affymetrix analysis, we can clearly see that, as Affymetrix releases more technical details of the chip design, researchers are developing alternative algorithms for this platform. 


\section{QA/QC IN MICROARRAYS}

A major goal in analyzing the Project Normal data set was to characterize two sources of variation: biological vs. technical. Using ANOVA modeling, both Xiangqin Cui and Russ Wolfinger pointed out that the technical variation of microarray hybridization is far larger than the biological variation of gene expression levels from mouse-to-mouse. Thus, technical variations have to be reduced to reveal true biological differences. Where do these technical variations arise? Lalush suggested a possible answer by looking at hybridization images. In his presentation at the preconference seminar, David Lockhart (Salk Institute) pointed out that there are many places in the microarray experiment process where QA/QC would be helpful to reduce the technical variation of experiments.

QA/QC is a standard industrial practice to ensure a quality product by controlling variances and detecting abnormalities during the manufacturing process. Many microarray practitioners from both industry and academia have the same concerns. George Wu, an attendee from UNC, Chapel Hill, noted that as the initial goldrush and enthusiasm for microarray screening has settled, concerns have been raised over the lack of reproducibility of control samples over time. Wu asks, "Can we comfortably put data together after we observe changes in the baseline control sample when measured a second time 6 months later?" An industry attendee, Xiaojun Guan from GlaxoSmithKline, said data quality becomes crucial to prevent error propagation as the pharmaceutical industry integrates proteomics and transcriptome data for drug discovery.

Since the 1980s, clinical chemistry laboratories have learned that the cost of production can be very expensive when there is insufficient emphasis on quality. Thus, $10 \%$ or more of consumable expenses have been allocated to QA/QC in clinical chemistry (7). In contrast, many microarray laboratories have only recently invested in QC protocols and practices. Holly Dressman (Duke University) noted that there are many experimental steps that are prone to error, yet easily corrected. For example, accidentally placing the slides upside-down when scanning the images can easily be rectified by careful laboratory procedures. Chen-Hsin Chen (Academia Sinica, Taiwan) said an effort is being made to standardize the operational procedures at different academic centers in Taiwan for microarray processing. Laura Reid (Expression Institute) pointed out that automatic sample handling and laboratory information systems (LIMS) are necessary to prevent human error, as the throughput and complexity of the analysis increases.

By the end of the conference, it was apparent that establishing industry standards for QA/QC is critical. A proposal to form a working group on QA and QC in microarray analysis was presented. Academic laboratories and industrial groups have expressed a willingness to work together to make recommendations for standard operation procedures for QA/QC. Several good preventive measures were discussed. John Quackenbush (The Institute for Genomic Research [TIGR]) noted that existing standard operating procedures (SOPs) are published at the TIGR Web site (www.tigr.org). In addition to preventive measures, Ronald Pearson (Thomas Jefferson University) discussed the use of outlier detection in microarray data. Coombes suggested using the parameters from Lalush's work for image quali- ty analysis. Patrick McConnell and Simon Lin (Duke University) are working on a spot checker program to analyze the quality of slide printing. Youxiang Wang (Fullmoon Biosystems) suggested using a scanner calibration slide as a tool to convert fluorescent signals of each spot to the number of dye molecules per square micron. Therefore, the variations from scanners can be eliminated, and the data obtained from different laboratories can be compared. Xiangqin Cui (Jackson Lab) contributed slides to show how a simple script can detect misalignment of the grids. It is hoped that the CAMDA QA/QC discussions will stimulate guidelines to generate quality microarray data, thus increasing the usefulness of combined databases.

\section{OTHER PRESENTATIONS}

In addition to data quality issues, there were also presentations of new analytical methods. Michael Ochs presented a Bayesian decomposition method to explain the patterns observed in different tissues, while Ramón Diaz-Uriarte (CNIO, Spain) investigated the utility of gene ontology for biological relevancies.

As in the past, the CAMDA conference provided a unique forum for discussions of microarray data analysis techniques. A list of the presentations at CAMDA'02 can be found at (www.camda.duke.edu), with abstracts and slides where available. The proceedings of CAMDA'02 will be published by Kluwer in early 2003, and the next CAMDA conference will be held in November, 2003. CAMDA'03 conference data sets are scheduled to be released in March. The CAMDA Web site provides updated information on all aspects of CAMDA with a mailing list option for notifications of important events.

\section{REFERENCES}

1.Spellman, P.T., M. Miller, J. Stewart, C. Troup, U. Sarkans, S. Chervitz, D. Bernhart, G. Sherlock, et al. 2002. Design and implementation of microarray gene expression markup language (MAGE-ML). Genome Biol. 3:RESEARCH0046.

2.Brazma, A., A. Robinson, G. Cameron, and M. Ashburner. 2000. One-stop shop for microarray data. Nature 403:699-700.

3.Pritchard, C.C., L. Hsu, J. Delrow, and P.S. Nelson. 2001. Project normal: defining normal variance in mouse gene expression. Proc. Natl. Acad. Sci. USA 98:13266-13271.

4.Wheeler, D.L., D.M. Church, A.E. Lash, D.D. Leipe, T.L. Madden, J.U. Pontius, G.D. Schuler, L.M. Schriml, et al. 2002. Database resources of the National Center for Biotechnology Information: 2002 update. Nucleic Acids Res. 30:13-16.

5.Li, C. and W.H. Wong. 2001. Model-based analysis of oligonucleotide arrays: expression index computation and outlier detection. Proc. Natl. Acad. Sci. USA 98:31-36.

6.Affymetrix. 2002. New statistical algorithms for monitoring gene expression on GeneChip ${ }^{\circledR}$ probe arrays. In Affymetrix Technical Note. Affymetrix, Santa Clara.

7.Cembrowski, G.S. and R.N. Carey. 1989. Laboratory quality management: $\mathrm{QC}$ and QA. American Society of Clinical Pathologists Press, Chicago.

Address correspondence to:

Dr. Simon M. Lin

Duke Bioinformatics Shared Resource

Duke University Medical Center

Box 3958

Durham, NC 27710, USA

e-mail:lin00025@mc.duke.edu 